\title{
REFLEXIVITY OF RINGS VIA NILPOTENT ELEMENTS
}

\author{
ABDULLAH HARMANCI, HANDAN KOSE, YOSUM KURTULMAZ, AND BURCU UNGOR
}

\begin{abstract}
An ideal $I$ of a ring $R$ is called left $N$-reflexive if for any $a \in$ $\operatorname{nil}(R)$ and $b \in R, a R b \subseteq I$ implies $b R a \subseteq I$, where $\operatorname{nil}(R)$ is the set of all nilpotent elements of $R$. The ring $R$ is called left $N$-reflexive if the zero ideal is left N-reflexive. We study the properties of left N-reflexive rings and related concepts. Since reflexive rings and reduced rings are left N-reflexive rings, we investigate the sufficient conditions for left $\mathrm{N}$-reflexive rings to be reflexive and reduced. We first consider basic extensions of left N-reflexive rings. For an ideal-symmetric ideal $I$ of a $\operatorname{ring} R, R / I$ is left N-reflexive. If an ideal $I$ of a ring $R$ is reduced as a ring without identity and $R / I$ is left N-reflexive, then $R$ is left N-reflexive. If $R$ is a quasi-Armendariz ring and the coefficients of any nilpotent polynomial in $R[x]$ are nilpotent in $R$, it is proved that $R$ is left $\mathrm{N}$-reflexive if and only if $R[x]$ is left $\mathrm{N}$-reflexive. We show that the concept of left N-reflexivity is weaker than that of reflexivity and stronger than that of right idempotent reflexivity.
\end{abstract}

\section{INTRODUCTION}

Throughout this paper, all rings are associative with identity. A ring is called reduced if it has no nonzero nilpotent elements. A weaker condition than reduced is defined by Lambek in [16]. A ring $R$ is said to be symmetric if for any $a, b, c \in R$, $a b c=0$ implies $a c b=0$. Equivalently, $a b c=0$ implies $b a c=0$. It is easily checked that if $R$ is a reduced ring, then the following condition holds: $a b=0$ implies $b a=0$ for any $a, b \in R$. Cohn [7] called a ring $R$ reversible if this condition holds. Anderson and Camillo [3] studied the rings whose zero products commute, and used the term ZC2 for what is called reversible. Prior to Cohn's work, reversible rings were studied under the names of completely reflexive and zero commutative by Mason [17] and Habe [8, respectively. Tuganbaev [18] investigated reversible rings under the name of commutative at zero. It is obvious that commutative rings and reduced rings are reversible. The reversible property of a ring is generalized as: A ring $R$ is said to satisfy the commutativity of nilpotent elements at zero ([2, Definition 2.1]) if $a b=0$ for any $a, b \in \operatorname{nil}(R)$ implies $b a=0$; for simplicity, such a ring is called $C N Z$.

2010 Mathematics Subject Classification. 16N40, 16S80, 16S99, 16 U80.

Key words and phrases. Reflexive ring; left N-reflexive ring; left N-right idempotent reflexive ring; quasi-Armendariz ring; nilpotent element. 
In [17, a right ideal $I$ of $R$ is said to be reflexive if $a R b \subseteq I$ implies $b R a \subseteq I$ for any $a, b \in R$. A ring $R$ is called reflexive if 0 is a reflexive ideal of $R$. Reversible rings are reflexive by [14, Proposition 2.2]. In [19, $R$ is said to be a weakly reflexive ring if $a R b=0$ implies $b R a \subseteq \operatorname{nil}(R)$ for any $a, b \in R$. In [13, a ring $R$ is said to be nil-reflexive if $a R b \subseteq \operatorname{nil}(R)$ implies that $b R a \subseteq \operatorname{nil}(R)$ for any $a, b \in R$. In [1, $R$ is called a reflexivity with maximal ideal axis ring (an $R M$ ring, for short) if for a maximal ideal $M$ and for any $a, b \in R, a M b=0$ implies $b M a=0$; similarly, $R$ has reflexivity with maximal ideal axis on idempotents (simply, $R M I$ ) if eMf $=0$ for any idempotents $e, f$ and a maximal ideal of $M$ yields $f M e=0$. In [15], $R$ has reflexive-idempotents-property (simply, RIP) if $e R f=0$ for any idempotents $e, f$ yields $f R e=0$. A left ideal $I$ is called idempotent reflexive [11] if aRe $\subseteq I$ implies $e R a \subseteq I$ for $a, e^{2}=e \in R$. A ring $R$ is called idempotent reflexive if 0 is an idempotent reflexive ideal. Kim and Baik [12 introduced the left and right idempotent reflexive rings. A two sided ideal $I$ of a $\operatorname{ring} R$ is called right idempotent reflexive if $a R e \subseteq I$ implies $e R a \subseteq I$ for any $a, e^{2}=e \in R$. A ring $R$ is called right idempotent reflexive if 0 is a right idempotent reflexive ideal. Left idempotent reflexive ideals and rings are defined similarly. If a ring $R$ is left and right idempotent reflexive, then it is called an idempotent reflexive ring.

Kheradmand et al. 10 generalized the notion of reflexive rings to RNP rings. A ring $R$ is called $R N P$ (reflexive-nilpotents-property) if $a R b=0$ for any $a, b \in$ $\operatorname{nil}(R)$ implies $b R a=0$. In this paper, motivated by these classes of types of reflexive rings, we introduce left $\mathrm{N}$-reflexive rings and right $\mathrm{N}$-reflexive rings. We prove that some results of reflexive rings can be extended to the left N-reflexive rings for this general setting. We investigate characterizations of left N-reflexive rings and many families of left N-reflexive rings are presented. The concept of one-sided N-reflexivity for rings is placed between reflexive rings and RNP rings.

In what follows, $\mathbb{Z}$ denotes the ring of integers and for a positive integer $n$, $\mathbb{Z}_{n}$ is the ring of integers modulo $n$. We write $M_{n}(R)$ for the ring of all $n \times n$ matrices; $U(R)$, nil $(R)$ will denote respectively the group of units and the set of all nilpotent elements of $R ; U_{n}(R)$ is the ring of upper triangular matrices over $R$ for a positive integer $n \geq 2 ; D_{n}(R)$ is the ring of all matrices in $U_{n}(R)$ having main diagonal entries equal; and $V_{n}(R)$ is the subring of $U_{n}(R)$ described as: $V=\sum_{i=1}^{n} e_{i, i+1}$, where $e_{i, j}$ is the matrix unit having 1 in the $(i, j)$ entry and 0 elsewhere, $R V_{k}=\left\{r A \mid r \in R, A \in V_{k}\right\}$ for $k \in\{1, \ldots, n-1\}$, and $V_{n}(R)=R I_{n}+R V+\cdots+R V^{n-1}$ for a positive integer $n$.

\section{N-REFLEXIVITY OF RINGS}

In this section, we introduce some classes of rings, so-called left N-reflexive rings and right N-reflexive rings. These classes of rings generalize reflexive rings. We investigate which properties of reflexive rings hold for the left N-reflexive rings and right $\mathrm{N}$-reflexive rings. We supply an example to show that there are left N-reflexive rings that are neither right $\mathrm{N}$-reflexive nor reflexive nor reversible. It is shown that the class of left N-reflexive rings is closed under finite direct sums. We have an example to show that homomorphic images of a left $\mathrm{N}$-reflexive ring need not be 
left N-reflexive. Then we determine under what conditions a homomorphic image of a ring is left N-reflexive. We now give our main definition.

Definition 2.1. Let $R$ be a ring. An ideal $I$ of $R$ is called left $N$-reflexive if for any $a \in \operatorname{nil}(R)$ and $b \in R, a R b \subseteq I$ implies $b R a \subseteq I$. The ring $R$ is called left $N$-reflexive if the zero ideal is left N-reflexive. Similarly, $I$ is called right $N$-reflexive if for any $a \in \operatorname{nil}(R)$ and $b \in R, b R a \subseteq I$ implies $a R b \subseteq I$. The ring $R$ is called right $N$-reflexive if the zero ideal is right $\mathrm{N}$-reflexive. The ring $R$ is called $N$-reflexive if it is both left and right N-reflexive.

Every reflexive ring and every semiprime ring is N-reflexive. There are left Nreflexive rings which are neither semiprime nor reduced nor reversible. The concept of one-sided N-reflexivity for rings is placed between reflexive rings and RNP rings:

$\{$ reflexive rings $\} \subseteq\{$ one-sided N-reflexive rings $\} \subseteq\{\mathrm{RNP}$ rings $\}$.

These inclusions are strict. There are RNP rings that are not left N-reflexive and there are left N-reflexive rings that are not reflexive as shown in what follows.

Example 2.2. There are RNP rings that are not left N-reflexive.

Proof. Let $F$ be a field. Then $\operatorname{nil}\left(U_{2}(F)\right)=\left[\begin{array}{ll}0 & F \\ 0 & 0\end{array}\right]$. For any $A, B \in \operatorname{nil}\left(U_{2}(F)\right)$, it is obvious that $A U_{2}(F) B=0$ implies $B U_{2}(F) A=0$. So $U_{2}(F)$ is an RNP ring. Let $C=\left[\begin{array}{ll}0 & 1 \\ 0 & 0\end{array}\right] \in \operatorname{nil}\left(U_{2}(F)\right), D=\left[\begin{array}{ll}1 & 1 \\ 0 & 0\end{array}\right] \in U_{2}(F)$. Then $C D=0$ and $D C \neq 0$. Hence $R$ is not left N-reflexive.

Let $F$ be a field and $R=F[x]$ the polynomial ring over $F$ with $x$ an indeterminate. Let $\alpha: R \rightarrow R$ be a homomorphism defined by $\alpha(f(x))=f(0)$, where $f(0)$ is the constant term of $f(x)$. Let $D_{2}^{\alpha}(R)$ denote the skewtrivial extension of $R$ by $R$ and $\alpha$. So $D_{2}^{\alpha}(R)=\left\{\left[\begin{array}{cc}f(x) & g(x) \\ 0 & f(x)\end{array}\right] \mid f(x), g(x) \in R\right\}$ is a ring with componentwise addition of matrices and multiplication as follows:

$$
\left[\begin{array}{cc}
f(x) & g(x) \\
0 & f(x)
\end{array}\right]\left[\begin{array}{cc}
h(x) & t(x) \\
0 & h(x)
\end{array}\right]=\left[\begin{array}{cc}
f(x) h(x) & \alpha(f(x)) t(x)+g(x) h(x) \\
0 & f(x) h(x)
\end{array}\right] .
$$

There are left N-reflexive rings which are neither reflexive nor semiprime. The N-reflexive property of rings is not left-right symmetric.

Example 2.3. Let $D_{2}^{\alpha}(R)$ denote the skewtrivial extension of $R$ by $R$ and $\alpha$ as mentioned above. Then by [19, Example 3.5], $D_{2}^{\alpha}(R)$ is not reflexive. We show that $D_{2}^{\alpha}(R)$ is left N-reflexive. Note that $\operatorname{nil}\left(D_{2}^{\alpha}(R)\right)=\left\{\left[\begin{array}{cc}0 & f(x) \\ 0 & 0\end{array}\right] \mid f(x) \in R\right\}$. Let $A=\left[\begin{array}{cc}0 & f(x) \\ 0 & 0\end{array}\right] \in \operatorname{nil}\left(D_{2}^{\alpha}(R)\right)$ and $B=\left[\begin{array}{cc}h(x) & g(x) \\ 0 & h(x)\end{array}\right] \in D_{2}^{\alpha}(R)$. Assume that $A D_{2}^{\alpha}(R) B=0$. We may assume $f(x) \neq 0$. Then $A D_{2}^{\alpha}(R) B=0$ reveals that $h(x)=0$, and also $B D_{2}^{\alpha}(R) A=0$. Hence $D_{2}^{\alpha}(R)$ is left N-reflexive. Next we show that $D_{2}^{\alpha}(R)$ is not right N-reflexive. Let $A=\left[\begin{array}{cc}0 & f(x) \\ 0 & 0\end{array}\right]$ be nilpotent and 
$B=\left[\begin{array}{cc}x h(x) & g(x) \\ 0 & x h(x)\end{array}\right] \in D_{2}^{\alpha}(R)$, with both $f(x) \neq 0$ and $h(x) \neq 0$. By definitions, $B D_{2}^{\alpha}(R) A=0$. Since $x h(x) f(x) \neq 0, A D_{2}^{\alpha}(R) B=\left[\begin{array}{cc}0 & f(x) r(x) x h(x) \\ 0 & 0\end{array}\right] \neq 0$ for some $0 \neq r(x) \in R$. So $D_{2}^{\alpha}(R)$ is not right $\mathrm{N}$-reflexive. On the other hand, $\operatorname{nil}\left(D_{2}^{\alpha}(R)\right)$ is an ideal of $D_{2}^{\alpha}(R)$ and $\left(\operatorname{nil}\left(D_{2}^{\alpha}(R)\right)\right)^{2}=0$ but $\operatorname{nil}\left(D_{2}^{\alpha}(R)\right) \neq 0$. Therefore $D_{2}^{\alpha}(R)$ is not semiprime.

Proposition 2.4. Let $R$ be a left $N$-reflexive ring. Then for any idempotent e of $R$, eRe is also left $N$-reflexive.

Proof. Let eae $\in$ eRe be nilpotent and ebe $\in e R e$ an arbitrary element with eaeRebe $=0$. Then we have ebeReae $=0$, since $R$ is left N-reflexive.

For any positive integer $n$, the full matrix ring $M_{n}(F)$ over any field $F$ is $\mathrm{N}$ reflexive but $M_{n}(F)$ has some subrings neither left N-reflexive nor right N-reflexive, as shown below.

Examples 2.5. (1) Let $F$ be a field and $R=M_{n}(F)$. In fact, $R$ is a simple ring, therefore prime. Let $A, B \in R$ with $A R B=0$. Since $R$ is prime, $A=0$ or $B=0$. Hence $B R A=0$. So $R$ is reflexive. Therefore $R$ is N-reflexive.

(2) Let $F$ be a field and consider the subrings $U_{n}(F)$ and $D_{n}(F)$ of $M_{n}(F)$. It is obvious that these subrings are neither left N-reflexive nor right N-reflexive.

There are some subrings of $M_{n}(R)$ that are N-reflexive.

Proposition 2.6. Let $R$ be a commutative ring. Then $V_{n}(R)$ is an $N$-reflexive ring.

The commutativity of the ring $R$ in Proposition 2.6 is not superfluous.

Example 2.7. Let $R$ be a ring and consider the ring $S=V_{2}\left(U_{2}(R)\right)$. Note that $U_{2}(R)$ is not commutative. Let $A=e_{12}+e_{14}+e_{34} \in \operatorname{nil}(S)$ and $B=$ $e_{11}+e_{12}+e_{33}+e_{34} \in S$, where $e_{i, j}$ is the matrix unit having 1 in the $(i, j)$ entry and 0 elsewhere. Then $A S B=0$ and $B A \neq 0$. Hence $S$ is not left N-reflexive.

Lemma 2.8. N-reflexivity of rings is preserved under isomorphisms.

Theorem 2.9. Let $R$ be a ring and $n$ a positive integer. If $M_{n}(R)$ is left $N$ reflexive, then $R$ is left $N$-reflexive.

Proof. Suppose that $M_{n}(R)$ is a left N-reflexive ring. Let $e_{i j}$ denote the matrix unit whose $(i, j)$ entry is 1 and whose other entries are 0 . Then $R \cong R e_{11}=$ $e_{11} M_{n}(R) e_{11}$ is N-reflexive by Proposition 2.4 and Lemma 2.8

Proposition 2.10. Every reversible ring is $N$-reflexive.

The converse statement of Proposition 2.10 may not be true in general as shown below.

Example 2.11. By Examples 2.5 (1), $M_{2}(F)$ is both left and right N-reflexive. But it is not reversible. 
Theorem 2.12. Let $R$ be a ring. Then the following are equivalent:

(1) $R$ is left $N$-reflexive.

(2) $I R J=0$ implies $J R I=0$ for any ideal I generated by a nilpotent element and any nonempty subset $J$ of $R$.

(3) $I J=0$ implies $J I=0$ for any ideal $I$ generated by a nilpotent element and any ideal $J$ of $R$.

Proof. (1) $\Rightarrow(2)$ : Assume that $R$ is left N-reflexive. Let $I=R a R$ with $a \in R$ nilpotent and $0 \neq J \subseteq R$ such that $I R J=0$. Then for any $b \in J, a R b=0$. This implies that $b R a=0$, hence $b R(R a R)=b R I=0$ for any $b \in J$. Thus $J R I=0$.

$(2) \Rightarrow(3)$ : Let $I=R a R$ with $a \in R$ nilpotent and let $J$ be an ideal of $R$ such that $I J=0$. Then $J=R J$, so $I R J=0$. By $(2), J R I=0$, thus $J I=0$.

$(3) \Rightarrow(1)$ : Let $a \in R$ be nilpotent and $b \in R$ with $a R b=0$. Then $(R a R)(R b R)=$ 0 . By $(3),(R b R)(R a R)=0$. Hence $b R a=0$. Therefore $R$ is left N-reflexive.

For any element $a \in R, r_{R}(a)=\{b \in R \mid a b=0\}$ is called the right annihilator of a in $R$. The left annihilator of $a$ in $R$ is defined similarly and denoted by $l_{R}(a)$.

Proposition 2.13. Let $R$ be a ring. Then the following hold:

(1) $R$ is left $N$-reflexive if and only if for any nilpotent element a of $R, r_{R}(a R) \subseteq$ $l_{R}(R a)$.

(2) $R$ is right $N$-reflexive if and only if for any nilpotent element a of $R, l_{R}(R a) \subseteq$ $r_{R}(a R)$.

Proof. (1) For the necessity, let $x \in r_{R}(a R)$ for any nilpotent element $a \in R$. We have $(a R) x=0$. The ring $R$ being left N-reflexive implies $x R a=0$. So $x \in l_{R}(R a)$.

For the sufficiency, let $a \in \operatorname{nil}(R)$ and $b \in R$ with $a R b=0$. Then $b \in r_{R}(a R)$. By hypothesis, $b \in l_{R}(R a)$, and so $b R a=0$. Thus $R$ is left $\mathrm{N}$-reflexive.

(2) Similar to the proof of (1).

For a field $F, D_{3}(F)$ is neither left N-reflexive nor right $\mathrm{N}$-reflexive. But there are some subrings of $D_{3}(F)$ that are N-reflexive as shown below.

Proposition 2.14. Let $R$ be a reduced ring. Then the following hold:

(1) Consider the subring $S=\left\{\left[\begin{array}{lll}a & b & c \\ 0 & a & 0 \\ 0 & 0 & a\end{array}\right] \mid a, b, c \in R\right\}$ of $D_{3}(R)$. Then $S$ is $N$-reflexive.

(2) Let $S=\left\{\left[\begin{array}{lll}a & 0 & c \\ 0 & a & b \\ 0 & 0 & a\end{array}\right] \mid a, b, c \in R\right\}$ be a subring of $D_{3}(R)$. Then $S$ is $N$ reflexive.

Proof. (1) Let $A=\left[\begin{array}{lll}0 & b & c \\ 0 & 0 & 0 \\ 0 & 0 & 0\end{array}\right] \in S$ be any nonzero nilpotent element and $B=$ $\left[\begin{array}{lll}u & v & t \\ 0 & u & 0 \\ 0 & 0 & u\end{array}\right] \in S$. Assume that $A S B=0$. This implies $A B=0$, and so $b u=0$ and 
$c u=0$. For any $C=\left[\begin{array}{lll}x & y & z \\ 0 & x & 0 \\ 0 & 0 & x\end{array}\right] \in S, B C A=\left[\begin{array}{ccc}0 & u x b & u x c \\ 0 & 0 & 0 \\ 0 & 0 & 0\end{array}\right]$. The equalities $b u=c u=0$ imply $(u x b)^{2}=(u x c)^{2}=0$. Since $R$ is reduced, $u x b=u x c=0$. Then $B C A=0$. Hence $B S A=0$. Thus $R$ is left N-reflexive. A similar proof implies that $S$ is right N-reflexive.

(2) By [19, Proposition 2.2].

The condition of $R$ being reduced in Proposition 2.14 is not superfluous, as the following example shows.

Example 2.15. Let $F$ be a field and $R=F\langle a, b\rangle$ be the free algebra with noncommuting indeterminates $a, b$ over $F$. Let $I$ be the ideal of $R$ generated by $a R b$ and $a^{2}$. Consider the $\operatorname{ring} \bar{R}=R / I$. Identify $a$ and $b$ with their images in $\bar{R}$. Then $a \bar{R} b=0$. But $b \bar{R} a \neq 0$. Note that $\bar{R}$ is not reduced. Consider the ring $S=\left\{\left[\begin{array}{ccc}x & y & z \\ 0 & x & 0 \\ 0 & 0 & x\end{array}\right] \mid x, y, z \in \bar{R}\right\}$. Let $B=\left[\begin{array}{ccc}b & 1 & 1 \\ 0 & b & 0 \\ 0 & 0 & b\end{array}\right] \in S$ and $A=\left[\begin{array}{lll}0 & a & a \\ 0 & 0 & 0 \\ 0 & 0 & 0\end{array}\right] \in \operatorname{nil}(S)$. Then $A S B=0$ since $a \bar{R} b=0$. However, $B A \neq 0$ since $b a \neq 0$. Hence $S$ is not left N-reflexive.

The class of left (or right) N-reflexive rings is not closed under homomorphic images.

Example 2.16. Consider the rings $R$ and $\bar{R}=R / I$ in Example 2.15 where $I$ is the ideal of $R$ generated by $a R b$ and $a^{2}$. Then $R$ is reduced, hence left $N$-reflexive. Let $\bar{a}, \bar{b} \in \bar{R}$. Then $\bar{a} \overline{R b}=0$. But $\bar{b} \bar{R} \bar{a} \neq 0$. Hence $\bar{R}$ is not left N-reflexive.

Let $R$ be a ring and $I$ an ideal of $R$. Recall (see [6]) that $I$ is called idealsymmetric if $A B C \subseteq I$ implies $A C B \subseteq I$ for any ideals $A, B, C$ of $R$. In this vein, we mention the following result.

Proposition 2.17. Let $R$ be a ring and $I$ an ideal-symmetric ideal of $R$. Then $R / I$ is an $N$-reflexive ring.

Proof. Let $\bar{a}$ denote the image of $a \in R$ in $R / I$ under the natural homomorphism from $R$ onto $R / I$. Let $\bar{a} \in \operatorname{nil}(R / I)$ and $\bar{b} \in R / I$ with $\bar{a}(R / I) \bar{b}=0$. Then $a R b \subseteq I$. So $R(R a R)(R b R) \subseteq I$. By hypothesis, $R(R b R)(R a R) \subseteq I$. Therefore $b R a \subseteq I$, and so $\bar{b}(R / I) \bar{a}=0$. It means that $R / I$ is left N-reflexive. Similarly, it can be shown that $R / I$ is also right $\mathrm{N}$-reflexive.

Let $R$ be a ring and $I$ an ideal of $R$. In the short exact sequence $0 \rightarrow I \rightarrow$ $R \rightarrow R / I \rightarrow 0, I$ being N-reflexive (as a ring without identity) and $R / I$ being $\mathrm{N}$-reflexive need not imply that $R$ is N-reflexive. 
Example 2.18. Let $F$ be a field and consider the ring $R=D_{3}(F)$. Let $I=$ $\left[\begin{array}{ccc}0 & F & F \\ 0 & 0 & F \\ 0 & 0 & 0\end{array}\right]$. Then $I$ is left N-reflexive since $I^{3}=0$. Also, $R / I$ is left N-reflexive since $R / I$ is isomorphic to $F$. However, by Examples $2.5(2), R$ is not left Nreflexive.

Theorem 2.19. Let $R$ be a ring and $I$ an ideal of $R$. If $I$ is reduced as a ring (without identity) and $R / I$ is left $N$-reflexive, then $R$ is left $N$-reflexive.

Proof. Let $a \in \operatorname{nil}(R)$ and $b \in R$ with $a R b=0$. Then $\bar{a}(R / I) \bar{b}=0$ and $\bar{a} \in$ $\operatorname{nil}(R / I)$. By hypothesis, $\bar{b}(R / I) \bar{a}=0$. Hence $b R a \subseteq I$. Since $I$ is reduced and $b R a$ is nil, $b R a=0$.

Note that Example 2.18 shows also that the reduced condition on the ideal $I$ in Theorem 2.19 is not superfluous.

Let $R$ be a ring and $e$ an idempotent in $R$. Then $e$ is called left semicentral if $r e=e r e$ for all $r \in R$, and $S_{l}(R)$ denotes the set of all left semicentral elements. Similarly, $e$ is called right semicentral if er $=$ ere for all $r \in R$, and $S_{r}(R)$ denotes the set of all right semicentral elements of $R$. We use $B(R)$ for the set of central idempotents of $R$. In [5], a ring $R$ is called left (right) principally quasi-Baer (or simply, left (right) p.q.-Baer) if the left (right) annihilator of a principal left (right) ideal of $R$ is generated by an idempotent as a left (right) ideal.

Theorem 2.20. The following hold for a ring $R$ :

(1) If $R$ is right $N$-reflexive, then $S_{l}(R)=B(R)$.

(2) If $R$ is left $N$-reflexive, then $S_{r}(R)=B(R)$.

Proof. (1) Let $e \in S_{l}(R)$ and $a \in R$. Then $(1-e) R e=0$. It follows that $(1-$ $e) R e(a-a e)=(1-e) R(e a-e a e)=0$. Since $e a-e a e$ is nilpotent and $R$ is right N-reflexive, $(e a-e a e) R(1-e)=0$. Hence $(e a-e a e)(1-e)=0$. This implies $e a-e a e=0$. On the other hand, $(1-e) R(a-e a) e \subseteq(1-e) R e=0$. Thus $(1-e) R(a e-e a e)=0$, and so $(1-e)(a e-e a e)=0$. Then $a e-e a e=0$. So we have $e a=a e$, i.e., $e \in B(R)$. Therefore $S_{l}(R) \subseteq B(R)$. The reverse inclusion is obvious.

(2) Similar to the proof of (1).

Theorem 2.21. Let $R$ be a right p.q.-Baer ring. Then the following conditions are equivalent:

(1) $R$ is a semiprime ring.

(2) $S_{l}(R)=B(R)$.

(3) $R$ is a reflexive ring.

(4) $R$ is a right $N$-reflexive ring.

Proof. $(1) \Leftrightarrow(2)$ : By [5, Proposition 1.17(i)].

$(1) \Leftrightarrow(3)$ : By [14, Proposition 3.15].

$(3) \Rightarrow(4)$ : Clear by definitions.

$(4) \Rightarrow(2)$ : By Theorem 2.20 (1). 
Theorem 2.22. Let $R$ be a left p.q.-Baer ring. Then the following conditions are equivalent:

(1) $R$ is a semiprime ring.

(2) $S_{r}(R)=B(R)$.

(3) $R$ is a reflexive ring.

(4) $R$ is a left $N$-reflexive ring.

Proof. Similar to the proof of Theorem 2.21

Question: If a ring $R$ is N-reflexive, then is $R$ a 2-primal ring?

There is a 2-primal ring which is not N-reflexive.

Example 2.23. Consider the 2 by 2 upper triangular matrix ring $R=\left[\begin{array}{cc}\mathbb{Z}_{2} & \mathbb{Z}_{2} \\ 0 & \mathbb{Z}_{2}\end{array}\right]$ over the field $\mathbb{Z}_{2}$ of integers modulo 2. For $A=\left[\begin{array}{ll}0 & 1 \\ 0 & 0\end{array}\right] \in \operatorname{nil}(R)$ and $B=\left[\begin{array}{ll}1 & 1 \\ 0 & 0\end{array}\right] \in$ $R$, we have $A R B=0$ but $B R A \neq 0$. But $R$ is 2-primal by [4, Proposition 2.5].

Proposition 2.24. Let $\left\{R_{i}\right\}_{i \in I}$ be a class of rings. Then $R=\prod_{i \in I} R_{i}$ is left $N$ reflexive if and only if $R_{i}$ is left $N$-reflexive for each $i \in I$.

Proof. Assume that $R=\prod_{i \in I} R_{i}$ is left N-reflexive. By Proposition 2.4 for each $i \in I$ $R_{i}$ is left N-reflexive. Conversely, let $a=\left(a_{i}\right) \in R$ be nilpotent and $b=\left(b_{i}\right) \in R$ with $a R b=0$. Then $a_{i} R_{i} b_{i}=0$ for each $i \in I$. Since each $a_{i}$ is nilpotent in $R_{i}$ for each $i \in I$, by hypothesis, $b_{i} R_{i} a_{i}=0$ for every $i \in I$. Hence $b R a=0$. This completes the proof.

\section{EXTENSIONS OF N-REFLEXIVE RINGS}

In this section, we study some kinds of extensions of N-reflexive rings. We start with the Dorroh extension. The Dorroh extension $D(R, \mathbb{Z})=\{(r, n) \mid r \in R, n \in$ $\mathbb{Z}\}$ of a ring $R$ is a ring with operations $\left(r_{1}, n_{1}\right)+\left(r_{2}, n_{2}\right)=\left(r_{1}+r_{2}, n_{1}+n_{2}\right)$ and $\left(r_{1}, n_{1}\right)\left(r_{2}, n_{2}\right)=\left(r_{1} r_{2}+n_{1} r_{2}+n_{2} r_{1}, n_{1} n_{2}\right)$, where $r_{i} \in R$ and $n_{i} \in \mathbb{Z}$ for $i=1,2$.

Proposition 3.1. A ring $R$ is left $N$-reflexive if and only if the Dorroh extension $D(R, \mathbb{Z})$ of $R$ is left $N$-reflexive.

Proof. Firstly, we note that $\operatorname{nil}(D(R, \mathbb{Z}))=\{(r, 0) \mid r \in \operatorname{nil}(R)\}$. For the necessity, let $(a, b) \in D(R, \mathbb{Z})$ and $(r, 0) \in \operatorname{nil}(D(R, \mathbb{Z}))$ with $(r, 0) D(R, \mathbb{Z})(a, b)=0$. Then $(r, 0)(s, 0)(a, b)=0$ for every $s \in R$. Hence $r s\left(a+b 1_{R}\right)=0$ for all $s \in R$, and so $r R\left(a+b 1_{R}\right)=0$. Since $R$ is left N-reflexive, $\left(a+b 1_{R}\right) R r=0$. Thus $(a, b)(x, y)(r, 0)=\left(\left(a+b 1_{R}\right)\left(x+y 1_{R}\right) r, 0\right)=0$ for any $(x, y) \in D(R, \mathbb{Z})$. For the sufficiency, let $s \in R$ and $r \in \operatorname{nil}(R)$ with $r R s=0$. We have $(r, 0) \in \operatorname{nil}(D(R, \mathbb{Z}))$. This implies $(r, 0) D(R, \mathbb{Z})(s, 0)=0$. By hypothesis, $(s, 0) D(R, \mathbb{Z})(r, 0)=0$. In particular, $(s, 0)(x, 0)(r, 0)=0$ for all $x \in R$. Therefore $s R r=0$. So $R$ is left N-reflexive. 
Let $R$ be a ring and $S$ the subset of $R$ consisting of identity and central regular elements. Set $S^{-1} R=\left\{s^{-1} r \mid s \in S, r \in R\right\}$. Then $S^{-1} R$ is a ring with an identity.

Theorem 3.2. A ring $R$ is left $N$-reflexive if and only if $S^{-1} R$ is left $N$-reflexive.

Proof. Assume that $R$ is left N-reflexive and let $s^{-1} a \in \operatorname{nil}\left(S^{-1} R\right), t^{-1} b \in S^{-1} R$ with $\left(s^{-1} a\right)\left(S^{-1} R\right)\left(t^{-1} b\right)=0$. Then $a \in \operatorname{nil}(R)$ and $a R b=0$. By assumption, $b R a=0$. Hence $\left(t^{-1} b\right)\left(S^{-1} R\right)\left(s^{-1} a\right)=0$. This implies that $S^{-1} R$ is left N-reflexive. Conversely, assume that $S^{-1} R$ is left N-reflexive. Let $a \in \operatorname{nil}(R)$ and $b \in R$ with $a R b=0$. Since $1 \in S,(1 a)\left(S^{-1} R\right)(1 b)=0$. It follows that $(1 b)\left(S^{-1} R\right)(1 a)=0$. This yields $b R a=0$. Therefore $R$ is left N-reflexive.

Corollary 3.3. For a ring $R, R[x]$ is left $N$-reflexive if and only if $R\left[x ; x^{-1}\right]$ is left $N$-reflexive.

Proof. Consider the subset $S=\left\{1, x, x^{2}, \ldots\right\}$ of $R[x]$. Then $S$ consists of 1 and central regular elements. So the claim holds by Theorem 3.2

Proposition 3.4. For a ring $R, R[x]$ is left $N$-reflexive if and only if $\left(S^{-1} R\right)[x]$ is left $N$-reflexive.

Proof. For the necessity, let $R[x]$ be left N-reflexive, $f(x)=\sum_{i=0}^{m} s_{i}^{-1} a_{i} x^{i}$ nilpotent, and $g(x)=\sum_{i=0}^{n} t_{i}^{-1} b_{i} x^{i} \in\left(S^{-1} R\right)[x]$ such that $f(x)\left(S^{-1} R\right)[x] g(x)=0$. Let $s=s_{0} s_{1} \ldots s_{m}$ and $t=t_{0} t_{1} t_{2} \ldots t_{n}$. Then $f_{1}(x)=s f(x)$ is nilpotent, $g_{1}(x)=$ $\operatorname{tg}(x) \in R[x]$, and $f_{1}(x) R[x] g_{1}(x)=0$. By hypothesis, $g_{1}(x) R[x] f_{1}(x)=0$. Then $g(x)\left(S^{-1} R\right)[x] f(x)=0$. The sufficiency is clear.

According to 9, a ring $R$ is said to be quasi-Armendariz if whenever $f(x)=$ $\sum_{i=0}^{m} a_{i} x^{i}$ and $g(x)=\sum_{j=0}^{n} b_{j} x^{j} \in R[x]$ satisfy $f(x) R[x] g(x)=0$, we have $a_{i} R b_{j}=$ 0 for each $i, j$.

The left N-reflexivity or right N-reflexivity and the quasi-Armendariz property of rings do not imply each other.

Examples 3.5. (1) Let $F$ be a field and consider the $\operatorname{ring} R=\left[\begin{array}{cc}F & F \\ 0 & F\end{array}\right]$. Then $R$ is quasi-Armendariz by [9, Corollary 3.15]. However, $R$ is not left N-reflexive. For $A=\left[\begin{array}{ll}0 & 1 \\ 0 & 0\end{array}\right] \in \operatorname{nil}(R)$ and $B=\left[\begin{array}{ll}1 & 1 \\ 0 & 0\end{array}\right] \in R$, we have $A R B=0$ but $B A \neq 0$.

(2) Consider the ring $R=\left\{\left[\begin{array}{ll}a & b \\ 0 & a\end{array}\right] \mid a, b \in \mathbb{Z}_{4}\right\}$. Since $R$ is commutative, $R$ is N-reflexive. For $f(x)=\left[\begin{array}{ll}0 & 1 \\ 0 & 0\end{array}\right]+\left[\begin{array}{ll}2 & 1 \\ 0 & 2\end{array}\right] x$ and $g(x)=\left[\begin{array}{ll}0 & 1 \\ 0 & 0\end{array}\right]+\left[\begin{array}{ll}2 & 3 \\ 0 & 2\end{array}\right] x \in$ $R[x]$, we have $f(x) R g(x)=0$, and so by [9, Lemma 2.1] $f(x) R[x] g(x)=0$, but $\left[\begin{array}{ll}2 & 1 \\ 0 & 2\end{array}\right] R\left[\begin{array}{ll}0 & 1 \\ 0 & 0\end{array}\right] \neq 0$. Thus $R$ is not quasi-Armendariz.

Proposition 3.6. Let $R$ be a quasi-Armendariz ring. Assume that coefficients of any nilpotent polynomial in $R[x]$ are nilpotent in $R$. Then $R$ is left $N$-reflexive if and only if $R[x]$ is left $N$-reflexive. 
Proof. Suppose that $R$ is left N-reflexive and $f(x)=\sum_{i=0}^{m} a_{i} x^{i}, g(x)=\sum_{j=0}^{n} b_{j} x^{j} \in$ $R[x]$ with $f(x) R[x] g(x)=0$ and $f(x)$ nilpotent. The ring $R$ being quasi-Armendariz implies $a_{i} R b_{j}=0$ for all $i$ and $j$, and $f(x)$ being nilpotent gives rise to all $a_{0}$, $a_{1}, a_{2}, \ldots, a_{m}$ nilpotent. By supposition $b_{j} R a_{i}=0$ for all $i$ and $j$. Therefore $g(x) R[x] f(x)=0$, and so $R[x]$ is left N-reflexive. Conversely, assume that $R[x]$ is left N-reflexive. Let $a \in R$ be nilpotent and let $b \in R$ be any element with $a R b=0$. Then $a R[x] b=0$. Hence $b R[x] a=0$. Thus $b R a=0$ and $R$ is left N-reflexive.

Note that in the commutative case, the coefficients of any nilpotent polynomial are nilpotent. However, this is not the case for noncommutative rings in general. Therefore in Proposition 3.6 the assumption "coefficients of any nilpotent polynomial in $R[x]$ are nilpotent in $R$ " is not superfluous, as the following example shows.

Example 3.7. Let $S=M_{n}(R)$ for a ring $R$. Consider the polynomial $f(x)=$ $e_{21}+\left(e_{11}-e_{22}\right) x-e_{12} x^{2} \in S[x]$, where the $e_{i j}$ 's are the matrix units. Then $f(x)^{2}=0$, but $e_{11}-e_{22}$ is not nilpotent.

\section{Applications}

In this section, we study some subrings of full matrix rings whether or not they are left or right N-reflexive rings.

The rings $H_{(s, t)}(R)$ : Let $R$ be a ring and let $s, t$ be in the center of $R$. Let

$$
H_{(s, t)}(R)=\left\{\left[\begin{array}{lll}
a & 0 & 0 \\
c & d & e \\
0 & 0 & f
\end{array}\right] \in M_{3}(R) \mid a, c, d, e, f \in R, a-d=s c, d-f=t e\right\} .
$$

Then $H_{(s, t)}(R)$ is a subring of $M_{3}(R)$. Note that any element $A$ of $H_{(s, t)}(R)$ has the form $\left[\begin{array}{ccc}s c+t e+f & 0 & 0 \\ c & t e+f & e \\ 0 & 0 & f\end{array}\right]$.

Lemma 4.1. Let $R$ be a ring, and let $s, t$ be in the center of $R$. Then the set of all nilpotent elements of $H_{(s, t)}(R)$ is

$$
\operatorname{nil}\left(H_{(s, t)}(R)\right)=\left\{\left[\begin{array}{lll}
a & 0 & 0 \\
c & d & e \\
0 & 0 & f
\end{array}\right] \in H_{(s, t)}(R) \mid a, d, f \in \operatorname{nil}(R), c, e \in R\right\} .
$$

Proof. Let $A=\left[\begin{array}{lll}a & 0 & 0 \\ c & d & e \\ 0 & 0 & f\end{array}\right] \in \operatorname{nil}\left(H_{(s, t)}(R)\right)$ be nilpotent. There exists a positive integer $n$ such that $A^{n}=0$. Then $a^{n}=d^{n}=f^{n}=0$. Conversely, assume that $a^{n}=$ $0, d^{m}=0$, and $f^{k}=0$ for some positive integers $n, m, k$. Let $p=\max \{n, m, k\}$. Then $A^{2 p}=0$. 
Theorem 4.2. The following hold for a ring $R$ :

(1) If $R$ is a reduced ring, then $H_{(0,0)}(R)$ is $N$-reflexive but not reduced.

(2) If $R$ is reduced, then $H_{(1,0)}(R)$ is $N$-reflexive but not reduced.

(3) If $R$ is reduced, then $H_{(0,1)}(R)$ is $N$-reflexive but not reduced.

(4) $R$ is reduced if and only if $H_{(1,1)}(R)$ is reduced.

Proof. (1) Let $R$ be a reduced ring and $A=\left[\begin{array}{lll}a & 0 & 0 \\ c & a & e \\ 0 & 0 & a\end{array}\right] \in \operatorname{nil}\left(H_{(0,0)}(R)\right)$ be nilpotent. By Lemma $4.1 a$ is nilpotent. By assumption, $a=0$. Let $B=\left[\begin{array}{ccc}k & 0 & 0 \\ l & k & n \\ 0 & 0 & k\end{array}\right] \epsilon$ $H_{(0,0)}(R)$ with $A H_{(0,0)}(R) B=0$. Then $A B=0$ implies $c k=0$ and $e k=0$. For any $X=\left[\begin{array}{ccc}x & 0 & 0 \\ y & x & u \\ 0 & 0 & x\end{array}\right] \in H_{(0,0)}(R), A X B=\left[\begin{array}{ccc}0 & 0 & 0 \\ c x k & 0 & e x k \\ 0 & 0 & 0\end{array}\right]=0$. Then $c x k=0$ and $e x k=0$ for all $x \in R$. The ring $R$ being reduced implies $k x c=0$ and $k x e=0$ for all $x \in R$. Then $B X A=\left[\begin{array}{ccc}0 & 0 & 0 \\ k x c & 0 & k x e \\ 0 & 0 & 0\end{array}\right]=0$ for all $X \in H_{(0,0)}(R)$. Hence $H_{(0,0)}(R)$ is left N-reflexive. A similar discussion reveals that $H_{(0,0)}(R)$ is also right $\mathrm{N}$-reflexive. Note that $R$ being reduced does not imply $H_{(0,0)}(R)$ is reduced, because $A=\left[\begin{array}{lll}0 & 0 & 0 \\ 1 & 0 & 1 \\ 0 & 0 & 0\end{array}\right] \in H_{(0,0)}(R)$ is a nonzero nilpotent element.

(2) Let $R$ be a reduced ring, $A=\left[\begin{array}{lll}0 & 0 & 0 \\ 0 & 0 & e \\ 0 & 0 & 0\end{array}\right] \in \operatorname{nil}\left(H_{(1,0)}(R)\right)$, and $B=$ $\left[\begin{array}{ccc}f+c & 0 & 0 \\ c & f & d \\ 0 & 0 & f\end{array}\right] \in H_{(1,0)}(R)$, with $A H_{(1,0)}(R) B=0$. For any $C=\left[\begin{array}{ccc}m+n & 0 & 0 \\ n & m & u \\ 0 & 0 & m\end{array}\right] \in$ $H_{(1,0)}(R), A C B=0$. Then $e m f=0$ and $f m e=0$. This implies $B C A=0$. Therefore $H_{(1,0)}(R)$ is left N-reflexive. Similarly, $H_{(1,0)}(R)$ is also right N-reflexive.

(3) Assume that $R$ is a reduced ring and let $A=\left[\begin{array}{lll}0 & 0 & 0 \\ c & 0 & 0 \\ 0 & 0 & 0\end{array}\right] \in \operatorname{nil}\left(H_{(0,1)}(R)\right)$ and $B=\left[\begin{array}{ccc}e+f & 0 & 0 \\ a & e+f & e \\ 0 & 0 & f\end{array}\right] \in H_{(0,1)}(R)$, with $A H_{(0,1)}(R) B=0$. For any $C=$ $\left[\begin{array}{ccc}m+n & 0 & 0 \\ k & m+n & m \\ 0 & 0 & n\end{array}\right] \in H_{(0,1)}(R), A C B=0$. Then $c(m+n)(e+f)=0$ and $(e+f)(m+n) c=0$. This implies $B C A=0$. Therefore $H_{(0,1)}(R)$ is left N-reflexive. Similarly, $H_{(0,1)}(R)$ is also right N-reflexive. 
(4) Suppose that $R$ is a reduced ring and let $A=\left[\begin{array}{ccc}c+e+f & 0 & 0 \\ c & e+f & e \\ 0 & 0 & f\end{array}\right] \in$ $\operatorname{nil}\left(H_{(1,1)}(R)\right)$ be nilpotent. Then $f$ is nilpotent and so $f=0$. In turn, this implies $e=c=0$. Hence $A=0$. Conversely, assume that $H_{(1,1)}(R)$ is reduced. Let $a \in R$ with $a^{n}=0$. Let $A=\left[\begin{array}{lll}a & 0 & 0 \\ 0 & a & 0 \\ 0 & 0 & a\end{array}\right] \in H_{(1,1)}(R)$. Then $A$ is nilpotent. By assumption, $a=0$.

\section{Generalizhtions And some examples}

In this section, we introduce left N-right idempotent reflexive rings and right N-left idempotent reflexive rings, to generalize the reflexive idempotent rings in Kwak and Lee [14], Kim [11], and Kim and Baik [12]. We introduce the following classes of rings to produce counterexamples related to left N-reflexive rings. These classes of rings will be studied in detail in a subsequent paper by the authors.

Definition 5.1. Let $R$ be a ring. An ideal $I$ of $R$ is called left $N$-right idempotent reflexive if $a R e \subseteq I$ implies $e R a \subseteq I$ for any nilpotent $a \in R$ and $e^{2}=e \in R$. A ring $R$ is called left $N$-right idempotent reflexive if 0 is a left $\mathrm{N}$-right idempotent reflexive ideal. Right N-left idempotent reflexive ideals and rings are defined similarly. If a ring $R$ is left N-right idempotent reflexive and right N-left idempotent reflexive, then it is called an $\mathrm{N}$-idempotent reflexive ring.

Every left N-reflexive ring is a left N-right idempotent reflexive ring. But there are left N-right idempotent reflexive rings that are not left N-reflexive.

Examples 5.2. (1) Let $F$ be a field and $A=F\langle X, Y\rangle$ denote the free algebra generated by noncommuting indeterminates $X$ and $Y$ over $F$. Let $I$ denote the ideal generated by $Y X$. Let $R=A / I$ and let $x=X+I, y=Y+I \in R$. It is proved in [11, Example 5] that $R$ is abelian and so $R$ has reflexive-idempotents-property but not reflexive by showing that $x R y \neq 0$ and $y R x=0$. Moreover, $x y R x=0$ and $x R x y \neq 0$. This also shows that $R$ is not left N-reflexive since $x y$ is nilpotent in $R$.

(2) Let $F$ be a field and let $A=F\langle X, Y\rangle$ denote the free algebra generated by noncommuting indeterminates $X$ and $Y$ over $F$. Let $I$ denote the ideal generated by $X^{3}, Y^{3}, X Y, Y X^{2}, Y^{2} X$ in $A$. Let $R=A / I$ and let $x=X+I, y=Y+I \in R$. Then in $R, x^{3}=0, y^{3}=0, x y=0, y x^{2}=0, y^{2} x=0$. In [1, Example 2.3], $x R y=0, y R x \neq 0$, and idempotents in $R$ are 0 and 1 . Hence for any $r \in \operatorname{nil}(R)$ and $e^{2}=e \in R, r R e=0$ implies $e R r=0$. Thus $R$ is left N-right idempotent reflexive. We show that $R$ is not a left N-reflexive ring. Since any $r \in R$ has the form $r=k_{0}+k_{1} x+k_{2} x^{2}+k_{3} y+k_{4} y^{2}+k_{5} y x$ and $x$ is nilpotent, as noted above, $x R y=0$. However, $y R x \neq 0$ since $y x \neq 0$. Thus $R$ is not left $\mathrm{N}$-reflexive.

(3) Let $F$ be a field of characteristic zero and $A=F\langle X, Y, Z\rangle$ denote the free algebra generated by noncommuting indeterminates $X, Y$, and $Z$ over $F$. Let $I$ denote the ideal generated by $X A Y$ and $X^{2}-X$. Let $R=A / I$ and let $x=X+I$, $y=Y+I, z=Z+I \in R$. Then in $R, x R y=0$ and $x^{2}=x$. So $x y=0, y x$ 
is nilpotent, $x$ is idempotent, and $x R y x=0$. But $y x R x \neq 0$. Hence $R$ is not right N-left idempotent reflexive. In [14, Example 3.3], it is shown that $R$ is right idempotent reflexive.

\section{ACKNOWLEDGMENT}

The authors would like to thank the referee for the valuable suggestions and comments.

\section{REFERENCES}

[1] A. M. Abdul-Jabbar et al., Reflexivity with maximal ideal axes, Comm. Algebra 45 (2017), no. 10, 4348-4361. MR 3640812

[2] A. M. Abdul-Jabbar et al., On commutativity of nilpotent elements at zero, Commun. Korean Math. Soc. 32 (2017), no. 4, 811-826. MR 3731618

[3] D. D. Anderson and V. Camillo, Semigroups and rings whose zero products commute, Comm. Algebra 27 (1999), no. 6, 2847-2852. MR 1687281.

[4] G. F. Birkenmeier, H. E. Heatherly and E. K. Lee, Completely prime ideals and associated radicals, in Ring theory (Granville, OH, 1992), 102-129, World Sci. Publ., River Edge, NJ. MR 1344225

[5] G. F. Birkenmeier, J. Y. Kim and J. K. Park, Principally quasi-Baer rings, Comm. Algebra 29 (2001), no. 2, 639-660. MR 1841988

[6] V. Camillo, T. K. Kwak and Y. Lee, Ideal-symmetric and semiprime rings, Comm. Algebra 41 (2013), no. 12, 4504-4519. MR 3169534

[7] P. M. Cohn, Reversible rings, Bull. London Math. Soc. 31 (1999), no. 6, 641-648. MR 1711020

[8] J. M. Habeb, A note on zero commutative and duo rings, Math. J. Okayama Univ. 32 (1990), 73-76. MR 1112012

[9] Y. Hirano, On annihilator ideals of a polynomial ring over a noncommutative ring, J. Pure Appl. Algebra 168 (2002), no. 1, 45-52.|MR 1879930

[10] M. Kheradmand et al., Reflexive property restricted to nilpotents, J. Algebra Appl. 16 (2017), no. 3, 1750044, 20 pp. MR 3626711

[11] J. Y. Kim, Certain rings whose simple singular modules are GP-injective, Proc. Japan Acad. Ser. A Math. Sci. 81 (2005), no. 7, 125-128. MR 2172601.

[12] J. Y. Kim and J. U. Baik, On idempotent reflexive rings, Kyungpook Math. J. 46 (2006), no. 4, 597-601. MR 2282661

[13] H. Kose, B. Ungor and A. Harmanci, Nil-reflexive rings, Commun. Fac. Sci. Univ. Ank. Sér. A1 Math. Stat. 65 (2016), no. 1, 19-33. MR 3528878

[14] T. K. Kwak and Y. Lee, Reflexive property of rings, Comm. Algebra 40 (2012), no. 4, 15761594. MR 2913004

[15] T. K. Kwak and Y. Lee, Reflexive property on idempotents, Bull. Korean Math. Soc. 50 (2013), no. 6, 1957-1972. MR 3149574

[16] J. Lambek, On the representation of modules by sheaves of factor modules, Canad. Math. Bull. 14 (1971), 359-368. MR 0313324

[17] G. Mason, Reflexive ideals, Comm. Algebra 9 (1981), no. 17, 1709-1724. MR 0631884

[18] A. A. Tuganbaev, Semidistributive modules and rings, Mathematics and its Applications, 449, Kluwer Academic Publishers, Dordrecht, 1998. MR 1657183 
[19] L. Zhao, X. Zhu and Q. Gu, Reflexive rings and their extensions, Math. Slovaca 63 (2013), no. 3, 417-430. MR 3071963.

\author{
Abdullah Harmanci \\ Department of Mathematics, Hacettepe University, Ankara, Turkey \\ harmanci@hacettepe.edu.tr
}

Handan Kose

Department of Mathematics, Kirsehir Ahi Evran University, Kirsehir, Turkey

handan.kose@ahievran.edu.tr

Yosum Kurtulmaz

Department of Mathematics, Bilkent University, Ankara, Turkey yosum@fen.bilkent.edu.tr

Burcu Ungor ${ }^{\bowtie}$

Department of Mathematics, Ankara University, Ankara, Turkey

bungor@science. ankara.edu.tr

Received: February 22, 2019

Accepted: June 27, 2019 